\title{
The Importance of Routine Quality Control For Reproducible Pulmonary Measurements By in Vivo Micro-CT
}

\author{
Martina Mambrini \\ University of Parma \\ Laura Mecozzi \\ University of Parma \\ Erica Ferrini \\ University of Parma \\ Ludovica Leo \\ University of Parma \\ Davide Bernardi \\ University of Parma \\ Grandi Andrea \\ Chiesi (Italy) \\ Nicola Sverzellati \\ University of Parma \\ Livia Ruffini \\ Azienda Ospedaliero-Universitaria di Parma \\ Mario Silva \\ University of Parma \\ Franco Stellari ( $\square$ fb.stellari@chiesi.com ) \\ Chiesi (Italy)
}

\section{Research Article}

Keywords: Preclinical lung imaging, Micro-CT, Quality control, Lung segmentation, Longitudinal Monitoring

Posted Date: January 3rd, 2022

DOI: https://doi.org/10.21203/rs.3.rs-1194936/v1

License: (c) (i) This work is licensed under a Creative Commons Attribution 4.0 International License.

Read Full License 


\section{Abstract}

Micro-Computed Tomography (CT) imaging provides densitometric and functional assessment of lung diseases in animal models, playing a key role either in understanding disease progression or in drug discovery studies.

The generation of reliable and reproducible experimental data is strictly dependent on a system's stability. Quality Controls (QC) are essential to monitor micro-CT performance but, although QC procedures are standardized and routinely employed in clinical practice, detailed guidelines for preclinical imaging are lacking.

In this work, we propose a routine QC protocol for in vivo micro-CT, based on three commercial phantoms.

To investigate the impact of a detected scanner drift on image post-processing, a retrospective analysis using twenty-two healthy mice was performed and lung density histograms used to compare the Area Under Curve (AUC), the skewness and the kurtosis before and after the drift. As expected, statistically significant differences were found for all the selected parameters [AUC: $532 \pm 31$ vs. $420 \pm 38(p<0.001)$; skewness: $2.3 \pm 0.1$ vs. $2.5 \pm 0.1$ ( $p<0.001)$ and kurtosis: $4.2 \pm 0.3$ vs. $5.1 \pm 0.5(p<0.001)$ ], confirming the importance of the designed QC procedure to obtain a reliable longitudinal quantification of disease progression and drug efficacy evaluation.

\section{Introduction}

Computed Tomography (CT) represents one of the most relevant imaging technologies for the assessment of lung disorders in clinical practice. Since lung densitometry depends on X-Ray (XR) attenuation of pulmonary tissue, changes in lung tissue density can reflect parenchymal abnormalities providing a reproducible quantitative evaluation of the extent and severity of pulmonary diseases ${ }^{1-3}$.

The miniaturized version of CT (i.e. micro-CT) represents a powerful non-invasive tool in preclinical research for understanding the pathogenesis and dynamics of lung disorders in several animal models ${ }^{4-}$ 8 .

Histogram-based analyses are used to discriminate between aeration levels, identifying variations in lung density to allow accurate longitudinal assessment of lung disease progression ${ }^{8,9}$ in compliance with the $3 R$ rules (Replacement, Reduction, Refinement). Moreover, the integration of micro-CT technology in antifibrotic drug discovery can be useful for investigating drug efficacy in preclinical settings ${ }^{10-13}$. However, timing, reliability and reproducibility of the data generated by micro-CT in preclinical studies present crucial issues in characterizing the human disease and profiling the putative drug candidate for a move into the clinic. Although standardized protocols for image acquisition and anesthesia are adopted to generate accurate and reproducible datasets ${ }^{14}$, other factors can potentially undermine either the intraor the inter- experiment reproducibility of results. 
The implementation of Quality Control (QC) protocols is therefore essential in order to constantly monitor scanner performance: scan quality may be altered by instrumental drift, thus affecting image postprocessing ${ }^{15}$ and the interpretation of CT results, especially when a fully automatic analysis of CT scans is used $^{16}$.

Standardized quality procedures are employed for clinical CT systems to optimize patient safety and the reliability of densitometric data, to ascertain that image quality requirements are met and to prevent system drift over time. According to clinical requirements, daily and monthly QC protocols are applied based on dedicated CT phantoms ${ }^{17-19}$, beyond the requirements of annual maintenance ${ }^{20-24}$.

In contrast, only general guidelines are available for preclinical micro-CT scanners ${ }^{25}$, which leave aside longitudinal monitoring and focus on validating home-made CT imaging systems ${ }^{26}$ or characterizing their performance in terms of image quality, stability and X-ray delivered dose during acquisition ${ }^{27-29}$. Additionally, in-house-developed devices and protocols ${ }^{15,30,31}$ are not easily applicable to other imaging centres.

In this work, we outline a routine QC protocol for in vivo micro-CT, based on commercial preclinical phantoms, that is able to monitor over months the stability of the scanner to detect any drift and ensure reproducible longitudinal pulmonary measurement. The designed QC procedure is intended to be a detailed guideline, adaptable in its implementation for any micro-CT scanner.

\section{Materials And Methods}

Ad-hoc QC tests for micro-CT systems have been established, using three commercial imaging phantoms (QRM GmbH, Möhrendorf, Germany) (Table 1).

Phantoms are acquired by micro-CT monthly ${ }^{25-28,31}$ and segmentation maps with fixed regions of interest (ROIs) are applied to the scans for the assessment of the image quality parameters (Figure 1). These measurements are conducted in a grey levels scale, that corresponds to the original scale of CT scans after reconstruction.

The data reported in this study were collected on specific control charts and compared to baseline (BL) values, each defined by upper and lower tolerance limits, as suggested by literature ${ }^{25,31}$. BL values were initially established for our Quantum GX micro-CT (PerkinElmer, Inc. Waltham, MA), acquiring five consecutive scans of the $\mathrm{QC}$ phantoms ${ }^{31,32}$. For each monitored parameter, the $\mathrm{BL}$ and tolerance width were calculated, respectively:

$$
B L=\operatorname{Average}\left(\left(x_{j}\right)_{j=1, \ldots, 5}\right)
$$




$$
\text { Tolerancewidth }=2 * S D\left(\left(x_{j}\right)_{j=1, \ldots, 5}\right)
$$

in which $x$ represents an image quality parameter, $j$ is the index of weekly acquisition and SD is the standard deviation of the baseline measures. All measures obtained by the QC tests were then compared to the corresponding tolerance range (Table 2):

$$
\text { Tolerancerange }=B L \pm \text { Tolerancewidth }
$$

In this study, the dosimetry of the Quantum GX scanner ${ }^{33}$ and its impact on lung imaging were not objects of investigation, since they have been already evaluated in preclinical applications ${ }^{34-36}$, with findings suggesting that animal welfare was protected in this respect and that longitudinal CT data were not affected by multiple X-ray expositions.

\section{Micro-CT phantoms and corresponding QC tests.}

Water, Low Contrast and Bar Pattern phantoms (QRM GmbH, Möhrendorf, Germany) were used for quality control tests, as detailed below.

\subsection{Water phantom}

A cylindric device with a diameter of $32 \mathrm{~mm}$ and filled with milli-Q water, was employed to evaluate noise, to extract the absolute grey value for water and measure uniformity (Micro-CT water phantom, QRM $\mathrm{GmbH}$, Möhrendorf, Germany).

Noise test. The noise value was extracted applying a circular $\mathrm{ROI}\left(\mathrm{RO} \mathrm{I}_{\text {Noise }}\right)$ to five spatially contiguous reconstructed slices, along the longitudinal z-axis (Figure $1 \mathrm{~A})$. The ROI area $\left(80.4 \mathrm{~mm}^{2}\right)$ was chosen to be $10 \%$ of the cylinder base area $\left(16 \mathrm{~mm} \times 16 \mathrm{~mm} \times \pi=804 \mathrm{~mm}^{2}\right)$, according to IPEM and IEC indications ${ }^{37,38}$. The noise was defined as the SD of the water grey level (i.e. SD water) within the ROI (i.e. mean $\left.^{\text {water }} \pm S D^{\text {water }}\right)$. In order to obtain the monthly noise value, namely Noise, we calculated the average of the five $S D^{\text {water: }}$

Noise $=\sum_{i=1}^{5} \frac{\left(S D_{i}^{\text {water }}\right)}{5} \quad \mathrm{i}=$ slice index

Water evaluation. Water grey level was monitored applying the same ROI employed for the noise test (Figure $1 \mathrm{~A})$. In this case, we evaluated the mean water grey level value ( mean $_{\text {water }}$ ), averaging the values of the five contiguous cross sections that compose the ROls:

Water $=\sum_{i=1}^{5} \frac{\left(\text { meanvalue }_{i}^{\text {water }}\right)}{5} \quad \mathrm{i}=$ slice index 
Uniformity test. Five circular ROls were positioned in the centre and in peripheral locations of the image and propagated for five contiguous slices along z-axis, as shown in Figure 1B. The image quality parameter for the uniformity test, i.e. Uniformity, was calculated as the difference between the mean ${ }^{\text {water }}$ value of the central $\mathrm{ROI}_{\mathrm{a}}$ and the average of the four mean ${ }^{\text {water }}$ values of the peripheral ROls $\left(\mathrm{ROI}_{\mathrm{b}}, \mathrm{ROI}_{\mathrm{c}}, \mathrm{ROI}_{\mathrm{d}}, \mathrm{ROI}_{\mathrm{e}}\right)$ :

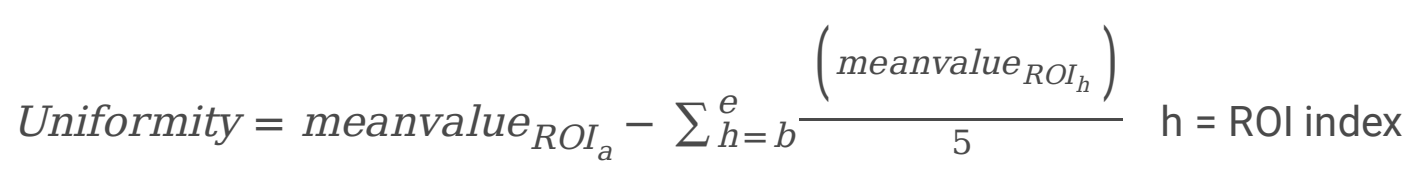

Each meanvalue ${ }_{R O I}$ was calculated by averaging the mean of water grey values obtained from the five contiguous slices, as in the 'Water evaluation' method.

\subsection{Micro-CT Low Contrast phantom}

The low contrast phantom was chosen to measure the contrast resolution of the scanner. It provides three approximate contrast levels of $-9 \%,-6 \%$ and $-3 \%$ compared to the background material, each level composed of three circular inserts with different diameters, i.e. 1, 2 and $3 \mathrm{~mm}$, for a total of nine inserts (Micro-CT Low Contrast Phantom V2, QRM GmbH, Möhrendorf).

Low contrast test. The low contrast detectability (LCD) is defined as the minimum visible dimension for low contrast objects, provided by the diameter of the smallest circular insert. The segmentation map for the LCD test consists of nine circular ROIs with dimensions proportional to the three different sizes of the inserts (Figure 1D). After adjusting the position of the ROls following the location of the inserts, the nine ROIs were propagated on 100 image slices to obtain a 3D evaluation. Two additional circular ROls were also considered: one for resin background and one for air (outside the phantom). The image quality parameter for the LCD test, called Contrast and expressed in \%, was calculated as follows:

$$
\text { Contrast }_{k}=\left[1-\frac{\left(\text { meanvalue }_{k}-\text { meanvalue }_{\text {air }}\right)}{\left(\text { meanvalue }_{\text {background }}-\text { meanvalue }_{\text {air }}\right)}\right] * 100
$$

where $k$ represents the insert index and ranges from one to nine $(k=1, \ldots, 9)$. Finally, for each contrast level $(-9 \%,-6 \%,-3 \%)$, the Contrast parameter was evaluated as the average value of the three inserts with the same low contrast level but different diameter sizes:

$$
\text { Contrast }^{c}=\left(\frac{\text { Contrast }_{1 m m}^{c}+\text { Contrast }_{2 m m}^{c}+\text { Contrast }_{3 m m}^{c}}{3}\right)
$$

in which the index $c$ can be $-9 \%,-6 \%$ or $-3 \%$. In this way, each month we obtained three values of Contrast, one for each nominal level.

\subsection{Micro-CT Bar Pattern phantom}


The spatial resolution test, as well as the evaluation of the absolute grey value for air, were carried out with the bar pattern phantom filled with air, and with a diameter of $20 \mathrm{~mm}$. Two chips placed inside the phantom contain bar patterns with different widths and points with different diameters allowed evaluation of the spatial resolution in the centre as well as in the periphery of the image in a single measurement (Micro-CT Bar Pattern Phantom, QRM GmbH, Möhrendorf).

Spatial resolution test. The in-plane spatial resolution for micro-CT images was tested. Based on visual inspection of the line pairs in the phantom scan (Figure 1E), the spatial resolution was in a range between 10 and 3.3 line pairs per $\mathrm{mm}(\mathrm{lp} / \mathrm{mm}$ ), corresponding to 50 to $150 \mu \mathrm{m}$ line width (Figure $1 \mathrm{~F}$ ), which represents the range in which the bars can be resolved ${ }^{26}$. The Modulation Transfer Function (MTF) of a system is used to describe the resolution performance of the scanner, providing a quantitative measure of the relationship between the original object and its radiological reconstruction ${ }^{39}$. MTF was calculated monthly for the three different bar patterns ( $3.3 \mathrm{lp} / \mathrm{mm}, 5 \mathrm{lp} / \mathrm{mm}$ and $10 \mathrm{lp} / \mathrm{mm})$ using the 'line profile' tool of Analyze software (Analyze 12.0; Copyright 1986-2017, Biomedical Imaging Resource, Mayo Clinic, Rochester, MN), https://www.analyzedirect.com. The value averaged over three measures for 'background' and 'bars' was recorded and MTF calculated as follow:

$$
\operatorname{MTF}^{C}=\left[\frac{\left(\text { meanvalue }_{\text {background }}-\text { meanvalue }_{\text {bars }}\right)}{\left(\text { meanvalue }_{\text {background }}+\text { meanvalue }_{\text {bars }}\right)}\right] * 100
$$

where the index c can be $3.3 \mathrm{lp} / \mathrm{mm}, 5 \mathrm{lp} / \mathrm{mm}$ or $10 \mathrm{lp} / \mathrm{mm}$. Since axial resolution is the same as transversal resolution, only the latter data are reported.

Air evaluation. The monitoring of grey levels of air was performed using a circular ROI positioned inside the bar pattern phantom (in air), with an area of $31.4 \mathrm{~mm}^{2}$, thus covering $10 \%$ of the phantom section area $\left(10 \mathrm{~mm} \times 10 \mathrm{~mm} \times \pi=314 \mathrm{~mm}^{2}\right)$. The ROI was propagated for five contiguous cross sections and the mean grey level of air $\left(\right.$ mean $\left._{\text {air }}\right)$ was calculated as the average of the five values extracted, as following:

Air $=\sum_{i=1}^{5} \frac{\left(\text { meanvalue air }_{i}^{\text {air }}\right)}{5} \quad \mathrm{i}=$ slice index

\section{Experimental control animals}

A cohort of 22 control mice were used for a retrospective evaluation of the impact of routine QC tests on lung scans post-processing.

Female inbred C57BI/6 (7- to 8- weeks old) mice were purchased from Envigo, Italy (San Pietro al Natisone, Udine, Italy). Prior to use, animals were acclimatized for at least 5 days to the local vivarium 
conditions (room temperature: $20-24^{\circ} \mathrm{C}$; relative humidity: $40-70 \%$; 12 -h light-dark cycle), having free access to standard rodent chow and softened tap water.

All animal experiments described herein were authorized by the official competent authority and approved by the intramural animal-welfare body (AWB) of Chiesi Farmaceutici and authorized by the Italian Ministry of Health (protocol number: 809/2020-PR). All procedures were conducted in an AAALAC (Association for Assessment and Accreditation for Laboratory Animal Care) certified facility in compliance with the European Directive 2010/63 UE, Italian D.Lgs 26/2014, the revised "Guide for the Care and Use of Laboratory Animals" 40 and with the Animal Research: Reporting of In Vivo Experiments (ARRIVE) guidelines ${ }^{41}$.

Animals were lightly anesthetized with $2.5 \%$ isoflurane delivered in a box and vehicle (50 $\mu \mathrm{l}$ saline [0.9\%]) was administered via oropharyngeal aspiration (OA) using a micropipette ${ }^{42}$. Twenty-one days after saline administration, mice were anesthetized with $2 \%$ isoflurane and underwent micro-CT imaging ${ }^{10,14}$.

\section{Micro-CT settings, images acquisition and processing}

The Quantum GX Micro-CT (PerkinElmer, Inc. Waltham, MA) was used in this study. This scanner has a microfocus X-ray source with a Tungsten anode. A fixed filter of $0.5 \mathrm{~mm}$ aluminium (Al) and $0.06 \mathrm{~mm}$ copper $(\mathrm{Cu})$ is placed in front of the exit port to remove low energy $\mathrm{X}$-rays. QC phantoms were acquired with the following parameters: X-ray tube voltage $90 \mathrm{KV}$, X-ray tube current $88 \mu \mathrm{A}$, total scan time of 4 min. A ring reduction correction was applied to the sinograms and the entire set of projection radiographs was input into a GPU-based filtered back-projection algorithm with a Ram-Lak filter. The acquisition protocol allows acquisition of projections over a total angle of $360^{\circ}$ resulting in $3 \mathrm{D}$ datasets with $50 \mu \mathrm{m}$ isotropic reconstructed voxel size $\mathrm{e}^{10,43}$. All the images were imported as $3 \mathrm{D}$.vox files and analysed using Analyze software (Analyze 12.0; Copyright 1986-2017, Biomedical Imaging Resource, Mayo Clinic, Rochester, MN) https://www.analyzedirect.com.

All protocols for mouse lung acquisition and image post-processing were largely detailed by Mecozzi et al. ${ }^{10}$ applied to a bleomycin-induced fibrosis murine model. Lung density histograms were extracted from pulmonary scans and the Area Under Curve (AUC), the skewness and kurtosis calculated.

\section{Statistical Analysis}

Statistical analyses were performed using GraphPad Prism version 9.1.2 for Windows (GraphPad Software, La Jolla, CA, USA), https ://www.graphpad.com. All data were presented as mean \pm SD. The normality test was performed for the AUC, the skewness and kurtosis of the average lung histograms. A one-tailed unpaired t-test was performed to compare AUC parameters. Finally, an unpaired t-test and Mann-Whitney test were performed to compare the kurtosis and the skewness, respectively. The alpha level of all tests was set at 0.05 .

\section{Results}


The grey level values of the QC parameters, monitored over 13 months, are reported in the control charts (Figure 2). For each test, BL appears as a continuous line, while dotted lines correspond to the upper and lower limits of the tolerance range.

The grey levels values for water (Figure 2A) and air (Figure 2B) remained stable within their tolerance ranges until the 12th month (blue squares). In the 13th month a shift towards higher levels was observed (red square), out of the upper limit of the tolerance ranges (2174 and 700 for water and air, respectively).

Also, noise and uniformity values (Figure $2 \mathrm{C}$ and 2D, respectively) were subjected to an analogous deviation from the $B L$ values in the 13th month; they reached 135 and -106 grey levels, respectively, thus deviating from the established tolerance ranges for noise $(122,126)$ and uniformity $(-63,-19)$.

Figure $3 A-C$, instead, shows the trends observed for the nominal contrast levels $-9 \%,-6 \%$ and $-3 \%$. The $B L$ values, measured for each contrast level, are different from the corresponding nominal value previously defined. We initially evaluated this discrepancy and agreed that it was acceptable for our purpose. Although the chosen acceptability ranges are narrow, the measurements performed in the 13th month were inside the tolerance limits for all the contrast levels. This means that during the whole observation period, the scanner was able to detect low contrast levels in lung scans, such as the tissue-air contrast ${ }^{26}$, despite the drift observed in the other parameters. The MTF for each of the three selected lp/mm bar patterns (i.e. spatial frequency) is reported in Figure 3D-F and expressed as a percentage (\%). These remained almost stable over the 13 months of evaluation and, overall, within acceptable values.

As a whole, as clearly depicted in Figure 2, the last point of observation (red square) in the control charts represents an out-of-control point for the majority of QC parameters: an increase in image noise, a decrease in image uniformity, along with strong fluctuations of water and air values towards higher grey levels.

To better investigate the effect of this scanner's drift on pulmonary scan post-processing and lung aeration levels, we performed a retrospective analysis on scans from 22 saline-treated mice, used as negative controls in lung fibrosis animal models. These animals were imaged during the period of investigation before (1st -12th months) and after the scanner's drift (13th month) and were included in the analysis.

The lungs of saline-treated mice are characterized by homogenous and well-aerated parenchyma. As expected, in the first period of observation, the lungs were entirely detectable and extracted using a semiautomatic segmentation protocol with well-defined thresholds ${ }^{10}$, as shown in Figure 4A (segmentation map) and Figure 4B (lung volume rendering). After the scanner drift, however, although experimental conditions, acquisition and image analysis protocols were unchanged, the usual segmentation protocol failed to detect the whole healthy parenchyma (Figure 4C-D). As outlined in the three-dimensional rendering (Figure 4D) some regions were excluded from the segmented map, even if they were sufficiently aerated to be detected and segmented. 
We also extracted the density histograms of the healthy lungs to assess the impact of the scanner's drift on image analysis. Figure $4 \mathrm{E}$ shows the average histograms, regarding the two periods before ( $1 \mathrm{st}-12 \mathrm{th}$ month: blue curve) and after (13th month: red curve) drift. The blue and red curves are averaged over 12 and 10 subjects, respectively. The shift of the red histogram towards higher grey levels could be linked to the concomitant increase in grey levels of water and air in QC scans detected during the 13th month. Using the established thresholds to segment lungs, we would wrongly generate incomplete lung maps, as detailed in Figure 4F. The AUC of the red histogram, which represents the total lung volume was, in fact, significantly smaller than the AUC of the blue histogram ( $532 \pm 31$ blue lung volume vs. $420 \pm 38$ red lung volume; $p<0.001)$. Moreover, both skewness ( $2.3 \pm 0.1$ blue vs. $2.5 \pm 0.1$ red; $p<0.001)$ and kurtosis ( 4.2 \pm 0.3 blue vs. $5.1 \pm 0.5$ red; $p<0.001$ ) parameters showed statistically significant differences, suggesting unjustified changes in the shape of the curves and the lung volumes of these healthy mice.

\section{Discussion And Conclusion}

In this work we demonstrated that changes in a micro-CT system can affect the quantitative analysis of $\mathrm{CT}$ images. Thus, a constant monitoring of micro-CT scanner performance is fundamental to recognizing instrumental drifts that can alter scan quality, impair image post-processing ${ }^{15}$ and corrupt the interpretation of CT results.

Although consistent guidelines for preclinical QC in micro-CT systems are still lacking, the definition of QC parameters and their tolerance ranges is a preliminary and very crucial step for the routine monitoring of such systems' performance. Starting from commercial QC phantoms we provided a standardized protocol for phantom acquisition and analysis. Contrary to clinical indications, which specifically define $\mathrm{BL} \pm 20 \%$ as the tolerance range for noise parameter, we found it more appropriate to consider $B L \pm 2 \star S D$ as the tolerance range for noise evaluation, as well as for the other QC parameters. This allowed identification of relevant fluctuations in some QC parameters, such as noise, uniformity and water/air grey levels, occurring as a result of a drift in the instrument.

We also demonstrated the importance of measuring the absolute grey levels of water and air during QC procedures, with the lung parenchyma being a soft tissue composed of tissue and air, and the latter representing its natural contrast. These two parameters have proven useful in detecting the shift in terms of grey levels which can affect micro-CT analysis ${ }^{15,30}$. In clinical practice, only Nowik et al. ${ }^{32}$ have suggested a protocol capable of monitoring the value of air during QC acquisition, but putting the ROI outside the phantom (contrary to the IEC recommendations), thus resulting in a highly variable estimation.

Overall, the variations we detected in the QC parameters acquired in the 13th month of observation suggest the need for a technical intervention. Probably, the worsening of the image quality, the increase of noise and the decrease of uniformity was caused by the deterioration of the aluminium-copper filter placed in front of the exit port of the source ${ }^{32}$. Among the parameters considered, these factors can 
directly influence the voxel value, the parameters related to the X-ray tube output and the reconstruction phase.

The retrospective analysis on data from healthy lungs confirmed that alterations in the micro-CT system (e.g. CT scanner reconstruction's algorithm and calibration, x-ray tube output, possible artefacts, etc.) impaired the post-processing phase. In pulmonary preclinical research, minor changes, if not immediately identified, negatively influence the lung segmentation process and the following densitometric quantification of disease progression or drug efficacy evaluation. For this reason, the ongoing monitoring of QC parameters is essential to ensure robust and reliable results during longitudinal studies, when the same animal is scanned weekly by micro-CT.

In addition, since the timing and the huge number of animals used in preclinical studies are leading to the integration of Artificial Intelligence models in pursuit of a completely standalone segmentation process ${ }^{44}$, the implementation of precise quality control procedures become mandatory.

To conclude, in this work we present a feasible and detailed operator-independent procedure for Quality Control of a micro-CT scanner, based on the acquisition of commercial QC phantoms, which any imaging facility could potentially integrate into their routine activities.

\section{Declarations}

\section{Authors' Contribution}

These authors contributed equally: Martina Mambrini and Laura Mecozzi.

Conception and design L.M., F.F.S. Laboratory testing E.F. Data collection L.M., M.M., E.F., L.L. Data analysis ad interpretation L.M., M.M., E.F., L.L., D. B, M.S., N.S, F.F.S. Drafting of Manuscript L.M., M.M, E.F., L.L., A.G., F.F.S. All authors contributed to the article and approved the submitted version.

\section{Competing interests}

The authors declare that this study received funding from Chiesi Farmaceutici S.p.A. The funder was not involved in the study design, data collection and analysis, decision to publish, or preparation of the manuscript.

F.F.S. and A.G. are employees of Chiesi Farmaceutici S.p.A., that supported the research work.

The remaining authors declare that the research was conducted in the absence of any commercial or financial relationships that could be construed as a potential conflict of interest.

\section{References}


1. Wu, X. et al. Computed tomographic biomarkers in idiopathic pulmonary fibrosis: The future of quantitative analysis. Am. J. Respir. Crit. Care Med. 199, 12-21 (2019).

2. Tielemans, B. et al. From Mouse to Man and Back: Closing the Correlation Gap between Imaging and Histopathology for Lung Diseases. Diagnostics 10, (2020).

3. Devaraj, Anand, Gianluca Milanese, and Nicola Sverzellati. 'Thoracic computed tomography in the progressive fibrotic phenotype.' Current Opinion in Pulmonary Medicine 27.5 (2021): 350-354.

4. Poelmans, J. et al. Longitudinal, in vivo assessment of invasive pulmonary aspergillosis in mice by computed tomography and magnetic resonance imaging. Lab. Investig. 96, 692-704 (2016).

5. Salaets, T. et al. Preterm birth impairs postnatal lung development in the neonatal rabbit model. Respir. Res. 21, 1-13 (2020).

6. Boudewijns, R. et al. STAT2 signaling restricts viral dissemination but drives severe pneumonia in SARS-CoV-2 infected hamsters. Nat. Commun. 11, 1-10 (2020).

7. Malengier-Devlies, B. et al. Lung Functioning and Inflammation in a Mouse Model of Systemic Juvenile Idiopathic Arthritis. Front. Immunol. 12, 1-13 (2021).

8. de Langhe, E. et al. Quantification of lung fibrosis and emphysema in mice using automated microcomputed tomography. PLoS One 7, (2012).

9. Dekoster, K. et al. Longitudinal micro-computed tomography-derived biomarkers quantify nonresolving lung fibrosis in a silicosis mouse model. Sci. Rep. 10, 1-10 (2020).

10. Mecozzi, L. et al. In - vivo lung fibrosis staging in a bleomycin - mouse model: a new micro - CT guided densitometric approach. Sci. Rep. 1-12 (2020) doi:10.1038/s41598-020-71293-3.

11. Ruscitti, F. et al. Quantification of Lung Fibrosis in IPF-Like Mouse Model and Pharmacological Response to Treatment by Micro-Computed Tomography. Front. Pharmacol. 11, 1-10 (2020).

12. Ruscitti, F., Ravanetti, F., Donofrio, G., Ridwan, Y., van Heijningen, P., Essers, J., Villetti, G., Cacchioli, A., Vos, W., Stellari, F. F. A Multimodal Imaging Approach Based on Micro-CT and Fluorescence Molecular Tomography for Longitudinal Assessment of Bleomycin-Induced Lung Fibrosis in Mice. J. Vis. Exp 134, (2018).

13. Ravanetti, F. et al. SSC-ILD mouse model induced by osmotic minipump delivered bleomycin: effect of Nintedanib. Sci. Rep. 11, 1-10 (2021).

14. Ferrini, E. et al. Alfaxalone and Dexmedetomidine as an Alternative to Gas Anesthesia for Micro-CT Lung Imaging in a Bleomycin-Induced Pulmonary Fibrosis Murine Model. Front. Vet. Sci. 7, 1-8 (2020).

15. Du, L. Y. et al. A quality assurance phantom for the performance evaluation of volumetric micro-CT systems. Phys. Med. Biol. 52, 7087-7108 (2007).

16. Birk, G., Kästle, M., Tilp, C., Stierstorfer, B. \& Klee, S. Automatization and improvement of $\mu$ CT analysis for murine lung disease models using a deep learning approach. 1-8 (2020).

17. De Oliveira, M. V. L., Wenzel, A., Campos, P. S. F. \& Spin-Neto, R. Quality assurance phantoms for cone beam computed tomography: A systematic literature review. Dentomaxillofacial Radiol. 46, (2017). 
18. Grimaud, J. \& Murthy, V. N. How to monitor breathing in laboratory rodents: a review of the current methods. J Neurophysiol. 2, 624-632 (2018).

19. Sumida, I. et al. Evaluation of imaging performance of megavoltage cone-beam CT over an extended period. J. Radiat. Res. 55, 191-199 (2014).

20. Moro, L. TOMOGRAFIA COMPUTERIZZATA: DESCRIZIONE E MISURA Traduzione, revisione e integrazione del documento : "Measurement of the Performance Characteristics of Diagnostic X-ray Systems used in Medicine". 4, (2007).

21. Dillon, C. et al. Computed Tomography Radiologist's Section Radiologic Technologist's Section Qualified Medical Physicist's Section QUALITY CONTROL MANUAL QUALITY CONTROL MANUAL Radiologist's Section Radiologic Technologist's Section Qualified Medical Physicist's Section. (2017).

22. EFOMP-ESTRO-IAEA. Quality control in cone-beam computed tomography (CBCT) EFOMP-ESTROIAEA protocol. 188 (2019).

23. Tradizionali, A., Radiografia, P. E. R., Mobili, F. E. \& Radioscopia, A. P. E. R. Cap. I Apparecchiature Tradizionali Per Radiografia Fisse E Mobili.

24. College of Physicians and Surgeons of British Columbia. Radiology and CT Quality Control Procedures Workbook. 1-134 (2018).

25. Osborne, D. R., Kuntner, C., Berr, S. \& Stout, D. Guidance for Efficient Small Animal Imaging Quality Control. Mol. Imaging Biol. 19, 485-498 (2017).

26. Preissner, M. et al. High resolution propagation-based imaging system for in vivo dynamic computed tomography of lungs in small animals. Phys. Med. Biol. 63, (2018).

27. Bretin, F. et al. Performance evaluation and $\mathrm{x}$-ray dose quantification for various scanning protocols of the GE explore 120 micro-ct. IEEE Trans. Nucl. Sci. 60, 3235-3241 (2013).

28. Khalil, M. M. Performance characteristics of the Inveon micro-CT scanner in small animal imaging. Biomed. Phys. Eng. Express 4, (2018).

29. Lee, Y. C., Fullerton, G. D., Baiu, C., Lescrenier, M. G. \& Goins, B. A. Preclinical multimodality phantom design for quality assurance of tumor size measurement. BMC Med. Phys. 11, 4-12 (2011).

30. Eloot, L. et al. Quality control of micro-computed tomography systems. Radiat. Prot. Dosimetry 139, 463-467 (2010).

31. Stoico, R., Tassani, S., Perilli, E., Baruffaldi, F. \& Viceconti, M. Quality control protocol for in vitro micro-computed tomography. J. Microsc. 238, 162-172 (2010).

32. Nowik, P., Bujila, R., Poludniowski, G. \& Fransson, A. Quality control of CT systems by automated monitoring of key performance indicators: A two-year study. J. Appl. Clin. Med. Phys. 16, 254-265 (2015).

33. Meganck, J. A. \& Liu, B. Dosimetry in Micro-computed Tomography: a Review of the Measurement Methods, Impacts, and Characterization of the Quantum GX Imaging System. Mol. Imaging Biol. 19, 499-511 (2017). 
34. Berghen, N. et al. Radiosafe micro-computed tomography for longitudinal evaluation of murine disease models. Sci. Rep. 9, 1-10 (2019).

35. Vande Velde, G. et al. Longitudinal in vivo microcomputed tomography of mouse lungs: No evidence for radiotoxicity. Am. J. Physiol. - Lung Cell. Mol. Physiol. 309, L271-L279 (2015).

36. Detombe, S. A., Dunmore-Buyze, J., Petrov, I. E. \& Drangova, M. X-ray dose delivered during a longitudinal micro-CT study has no adverse effect on cardiac and pulmonary tissue in C57BL/6 mice. Acta Radiologica vol. 54 435-441 (2013).

37. IEC. Evaluation and routine testing in medical imaging departments - Part 3-2: Acceptance tests Imaging performance of mammographic X-ray equipment. IEC Publ. (2007).

38. IEC. Evaluation and Routine Testing in Medical Imaging Departments: Part 2-6: Constancy Tests: Xray Equipment for Computed Tomography. I.S. En 61223-2-62007 p.1-11 (2007).

39. Boreman. Modulation Transfer Function in Optical and Electro-Optical Systems. (2001).

40. Bayne, K. Revised Guide for the Care and Use of Laboratory Animals available. American Physiological Society. The Physiologist vol. 39 (1996).

41. Percie Du Sert, N. et al. The ARRIVE guidelines 2.0: Updated guidelines for reporting animal research. BMC Vet. Res. 16, 1-7 (2020).

42. De Vooght V, Vanoirbeek JAJ, Haenen S, Verbeken E, Nemery B, H. P. Oropharyngeal aspiration: an alternative route for challenging in a mouse model of chemical-induced asthma. Toxicology 84-9. (2009).

43. Meganck, J. A. \& Liu, B. Dosimetry in Micro-computed Tomography: a Review of the Measurement Methods, Impacts, and Characterization of the Quantum GX Imaging System. Mol. Imaging Biol. 19, 499-511 (2017).

44. Walsh, S. L. F., Calandriello, L., Silva, M. \& Sverzellati, N. Deep learning for classifying fibrotic lung disease on high-resolution computed tomography: a case-cohort study. Lancet Respir. Med. 6, 837845 (2018).

\section{Tables}

Due to technical limitations, table $1 \& 2$ is only available as a download in the Supplemental Files section.

\section{Figures}



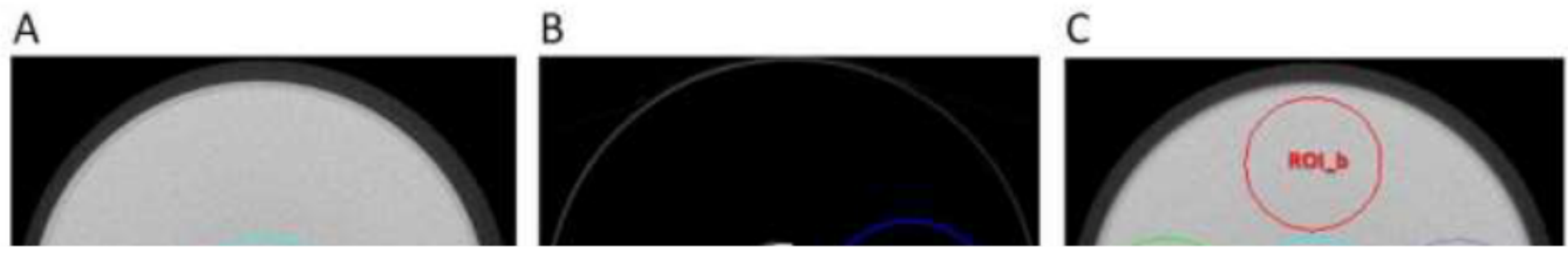

\section{Figure 1}

Axial views of micro-CT phantoms and segmentation maps used for the corresponding QC tests. (A) Water phantom with the ROI used to perform noise test and water value evaluation, within five contiguous slices. (B) Bar Pattern phantom filled with air with the ROI used to monitor air value, within five contiguous slices. (C) Water phantom with the ROls used for the uniformity test, within five contiguous slices: one central (ROI_a) and four peripheral ROIs (ROI_b, ROI_C, ROI_d, ROI_e). (D) Low Contrast V2 phantom: the cylindric inserts with different low contrast levels $(-9,-6$ and $-3 \%)$ are identified by means of proportional ROls, orange for $3 \mathrm{~mm}$ diameter, green for $2 \mathrm{~mm}$ diameter and blue for $1 \mathrm{~mm}$ diameter. The central yellow ROI is used to detect the background value. (E) The $5 \times 5 \mathrm{~mm}^{2}$ chip of the Bar Pattern phantom: the patterns used for the spatial resolution test are indicated in blue $(10 / \mathrm{p} / \mathrm{mm})$, red $(5 / \mathrm{p} / \mathrm{mm})$ and green $(3.31 \mathrm{p} / \mathrm{mm})$ and $(\boldsymbol{F})$ the corresponding line widths are reported. 

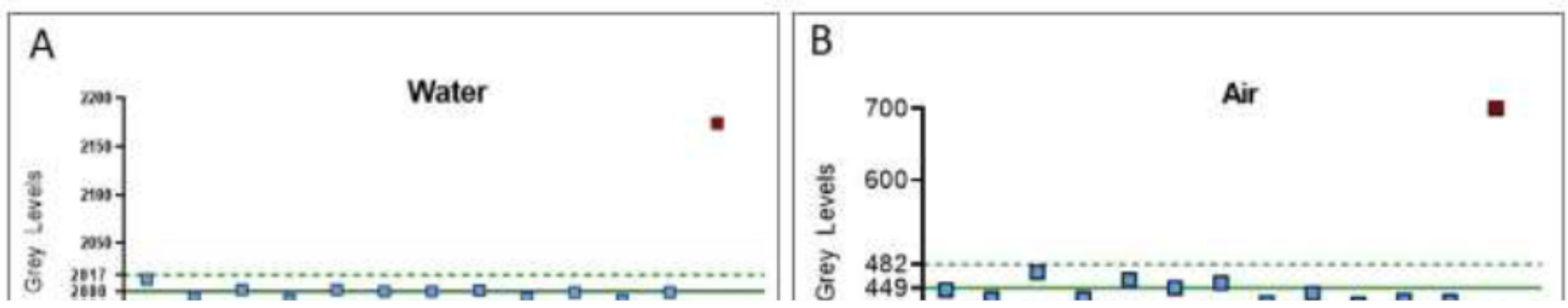

Figure 2

Control charts for Water and Air absolute grey levels (A-B) and Image noise and uniformity (C-D). The fluctuations of QC parameters were monitored during 13 months. BL values (continuous lines) and acceptability ranges (dotted lines) are highlighted in green for each control chart. 


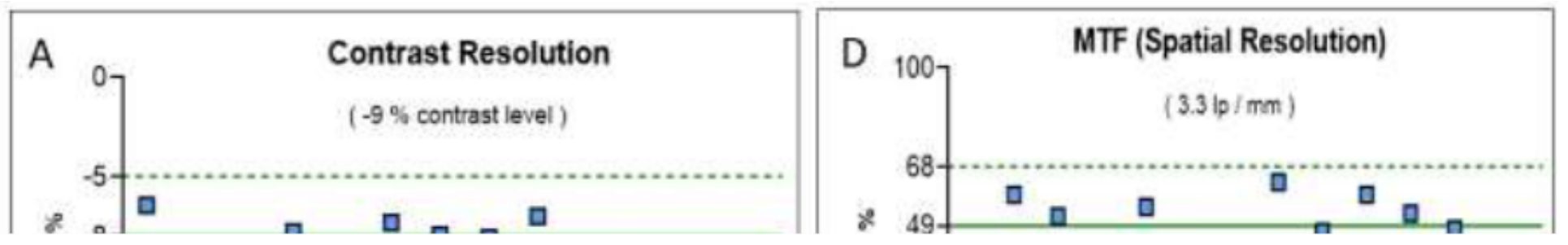

\section{Figure 3}

(A-B-C) Low contrast control charts for the known contrast levels of $-9 \%,-6 \%$ and $-3 \%$, respectively. (D-EF) Spatial resolution control charts, in terms of MTF\%, for the three different line pairs per $\mathrm{mm}$ patterns: $3.3 \mathrm{lp} / \mathrm{mm}, 5 \mathrm{lp} / \mathrm{mm}$ and $10 \mathrm{lp} / \mathrm{mm}$, respectively. The fluctuations of the QC parameters were monitored over 13 months. BL values (continuous lines) and acceptability ranges (dotted lines) are reported in green for each control chart. 


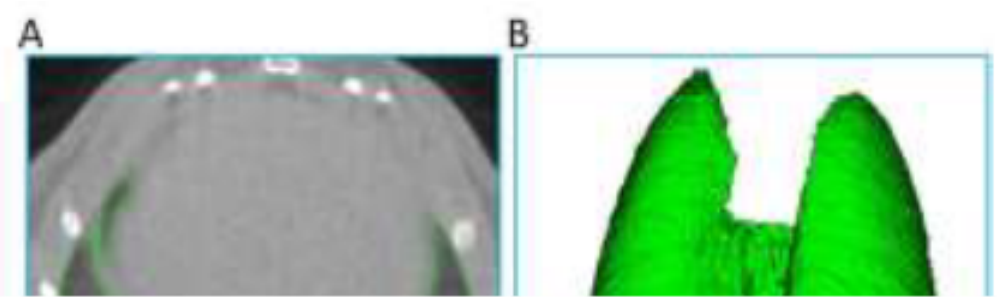

\section{Figure 4}

Effect of micro-CT scanner drift on image lung segmentation process. (A-B) A representative transversal slice of a saline mouse lung scan before the drift ( $1^{\text {st }}-12^{\text {th }}$ months): the lung segmentation map and the corresponding 3D rendering, obtained using an automatic segmentation, are highlighted in green. (C-D) A representative transversal slice and the corresponding $3 D$ rendering of a saline mouse lung scan from the $13^{\text {th }}$ month: the automatic segmentation fails to detect some healthy regions of aerated parenchyma 
(black arrows). (E) Lung mean grey levels density histograms before $\left(1^{\text {st }}-12^{\text {th }}\right.$ month: blue curve) and after ( $13^{\text {th }}$ month: red curve) drift. Each point of the histogram is represented as mean $\pm S D$. (F) Statistical comparison between the two average histograms in terms of AUC, Kurtosis and Skewness. All parameters are reported as mean $\pm S D$. The alpha level of statistical tests is set at $0.05\left({ }^{*} p<0.05,{ }^{*} p<0.01\right.$, $* * x<0.001)$.

\section{Supplementary Files}

This is a list of supplementary files associated with this preprint. Click to download.

- Table12.docx 